\title{
PRIME NONASSOCIATIVE RINGS WITH SIEW DERIVATIONS
}

\author{
CHEN-TE YEN
} \begin{abstract}
A bstract. Let $R$ be a prime nonassociative ring, $G$ the nucleus of $R$ and $s, t$ be automorphisms
of $R$.
(I) Suppose that $\delta$ is an $s$-derivation of $R$ such that $s \delta=\delta s$ and $\lambda$ is an $t$-derivation of $R$. If $\lambda \delta^{n}=0$ and $\delta^{n}(R) \subseteq G$, where $n$ is a fixed positive integer, then $\lambda=0$ or $\delta^{3 n-1}=0$.

(II) Assume that $\delta$ and $\lambda$ are derivations of $R$. If there exists a fixed positive integer $n$ such that $\lambda^{n} \delta=0$, and $\delta(R) \subseteq G$ or $\lambda^{n}(R) \subseteq G$, then $\delta^{2}=0$ or $\lambda^{6 n-4}=0$.
\end{abstract}

\section{Introduction}

Let $R$ be a nonassociative ring. We adopt the usual notation for commutators and associators: $[x, y]=x y-y x$ and $(x, y, z)=(x y) z-x(y z)$ for $x, y, z \in R$. We shall denote the nucleus of $R$ by $G$. Thus $G$ consists of all elements $n$ in $R$ such that $(n, R, R)=$ $(R, n, R)=(R, R, n)=0$. Denote the group of all automorphsisms of $R$ by $\operatorname{Aut}(R)$. An additive mapping $\delta$ from $R$ into $R$ is called a skew derivation or an $s$-derivation if $\delta(x y)=\delta(x) y+s(x) \delta(y)$ for all $x, y$ in $R$, where $s \in A u t(R)$. If $s$ is the identity automorphism of $R$ then $\delta$ is called a derivation of $R$. Let $\operatorname{Der}(R)$ be the Lie ring of derivations of $R$. A ring $R$ is called prime if the product of any two nonzero ideals of $R$ is nonzero.

Posner [3] proved that if $R$ is a prime associative ring of characteristic not two with derivations $\lambda$ and $\delta$ then $\lambda \delta \in \operatorname{Der}(R)$ implies $\lambda=0$ or $\delta=0$. Jensen [2] partially extended this result. Two of his results are as follows: If $R$ is a prime associative ring with derivations $\lambda, \delta$ and there exists a fixed positive integer $n$ such that $\lambda \delta^{n}=0\left(\lambda^{n} \delta=0\right)$ then $\lambda=0$ or $\delta^{4 n-1}=0\left(\delta^{2}=0\right.$ or $\left.\lambda^{12 n-9}=0\right)$. In this paper, we improve and generalize these results to the prime nonassociative rings with skew derivations.

In every ring $R$ we have the Teichmüller identity

$$
(\omega x, y, z)-(\omega, x y, z)+(\omega, x, y z)=\omega(x, y, z)+(\omega, x, y) z \text { for all } \omega, x, y, z \in R .
$$

Note that the associator $(x, y, z)$ is linear in each argument. Thus using (1), we have that $G$ is an associative subring of $R$.

Received February 21, 1997.

1991 Mathematics Subject Classification. Primary 17A36. Key words and phrases. Prime nonassociative ring, Nucleus, Skew derivation, Autompor-
phism.

This research is supported in part by the National Science Council, Republic of China under Grant
NSC 86-2115-M-033-009. 


\section{Results}

Our first main result is the following Theorem 1 , which improves and generalizes Jensen's Theorem 1.

Theorem 1. [5] Let $R$ be a prime nonassociative ring and let $s, t \in A$ A $(R)$. Suppose that $\delta$ is an $s$-derivation of $R$ such that $s \delta=\delta s$ and $\lambda$ is an $t$-derivation of $R$. If $\lambda \delta^{n}=0$ and $\delta^{n}(R) \subseteq G$, where $n$ is a fixed positive integer, then $\lambda=0$ or $\delta^{3 n-1}=0$.

To prove Theorem 1 we need a Lemma. The proof of the Lemma is the same as that of $[4$, lemma 1], except that the equation (4) of [4] is replaced by

$$
\delta^{n}(x y)=\sum_{i=0}^{n} d^{i}\left(s^{n-i}(x)\right) d^{n-i}(y) \in A .
$$

Lemma. Let $A$ be subring of $R$. If $\delta^{n}(R) \subseteq A$ then $R \delta^{3 n-1}(R) \subseteq A$. Proof of Theorem 1.

Let $\operatorname{ker}(\lambda)=\{c \in R: \lambda(c)=0\}$. Then $\operatorname{ker}(\lambda)$ is the subring of constants of $R$ under $\lambda$. The hypothesis $\lambda \delta^{n}=0$ implies $\delta^{n}(R) \subseteq \operatorname{ker}(\lambda)$. By the Lemma, we get $R \delta^{3 n-1}(R) \subseteq$ $\operatorname{ker}(\lambda)$. Since $\delta^{n}(R) \subseteq G$ and $s \delta=\delta s$, using [4, Theorem] we obtain that $R$ is associative or $\delta^{3 n-1}=0$. Assume that $\delta^{3 n-1} \neq 0$. Then $R$ is associative. Because of $R \delta^{3 n-1}(R) \subseteq$ $\operatorname{ker}(\lambda)$ and $\lambda \delta^{n}=0$, for all $x, y, z$ in $R$ we have

$$
0=\lambda\left(x \delta^{3 n-1}(y)\right)=\lambda(x) \delta^{3 n-1}(y)+t(x) \lambda\left(\delta^{3 n-1}(y)\right)=\lambda(x) \delta^{3 n-1}(y)
$$

and so $\lambda(z x) \delta^{3 n-1}(y)=0$. The last two equalities imply

$$
\begin{aligned}
0 & =(\lambda(z) x+t(z) \lambda(x)) \delta^{3 n-1}(y) \\
& =\lambda(z) x \delta^{3 n-1}(y)+t(z) \lambda(x) \delta^{3 n-1}(y) \\
& =\lambda(z) x \delta^{3 n-1}(y)
\end{aligned}
$$

By the primeness of $R$, this implies $\lambda(z)=0$ or $\delta^{3 n-1}(y)=0$. In view of $\delta^{3 n-1} \neq 0$, we obtain $\lambda(z)=0$ for all $z$ in $R$. Thus $\lambda=0$, as desired.

Our second main result is the following Theorem 2 , which improves and generalizes Jensen's Theorem 2.

Theorem 2. Let $R$ be a prime nonassociative ring and let $\delta$ and $\lambda$ be derivations of $R$. If there exists a fixed positive integer $n$ such that $\lambda^{n} \delta=0$, and $\delta(R) \subseteq G$ or
$\lambda^{n}(R) \subseteq G$, then $\delta^{2}=0$ or $\lambda^{6 n-4}=0$.

Proof. The derivations of $R$ form a Lie ring under commutation. Therefore $[\delta, \lambda]=$ $\delta \lambda-\lambda \delta$ is a derivation, $[\delta \lambda-\lambda \delta, \lambda]=\delta \lambda^{2}-2 \lambda \delta \lambda+\lambda^{2} \delta$ is a derivation, and $\left[\delta \lambda^{2}-2 \lambda \delta \lambda+\right.$ 

$\left.\lambda^{2} \delta, \lambda\right]=\delta \lambda^{3}-3 \lambda \delta \lambda^{2}+3 \lambda^{2} \delta \lambda-\lambda^{3} \delta$ is also a derivation. Continuing we may conclude
that

$$
\sum_{i=0}^{2 n-1}\left(\begin{array}{c}
2 n-1 \\
i
\end{array}\right)(-1)^{i} \lambda^{i} \delta \lambda^{2 n-1-i}
$$

is a derivation. The coefficients are not germane to the rest of the proof, so we suppress them from here on out. Thus, using $\lambda^{n} \delta=0$ we have that

$$
\delta \lambda^{2 n-1}+\lambda \delta \lambda^{2 n-2}+\cdots+\lambda^{n-1} \delta \lambda^{n} \text { is a derivation of } R \text {. }
$$

Since $\delta(R) \subseteq G$ or $\lambda^{n}(R) \subseteq G$, applying [4, Theorem ] we obtain that $R$ is associative, or $\delta^{2}=0$ or $\lambda^{3 n-1}=0$. If $\delta^{2}=0$ or $\lambda^{3 n-1}=0$, then we are done.

Suppose that $\delta^{2} \neq 0$ and $\lambda^{3 n-1} \neq 0$. Then $R$ is associative. Because of $\lambda^{n} \delta=0$, we get $\left(\delta \lambda^{2 n-1}+\lambda \delta \lambda^{2 n-1}+\cdots+\lambda^{n-1} \delta \lambda^{n}\right) \delta=0$. In view of $\delta^{2} \neq 0$, by (2) and Theorem 1
the last equality implies

$$
\delta \lambda^{2 n-1}+\lambda \delta \lambda^{2 n-2}+\cdots+\lambda^{n-1} \delta \lambda^{n}=0 .
$$

Premultiplying (3) by $\lambda^{n-1}$ and applying $\lambda^{n} \delta=0$, we obtain

$$
\lambda^{n-1} \delta \lambda^{2 n-1}=0 .
$$

Using (4) and premultiplying (3) by $\lambda^{n-2}$, it follows that $\lambda^{n-2} \delta \lambda^{2 n-1}+\lambda^{n-1} \delta \lambda^{2 n-2}=0$. Hence, we have $0=\left(\lambda^{n-2} \delta \lambda^{2 n-1}+\lambda^{n-1} \delta \lambda^{2 n-2}\right) \lambda$ and so by (4)

$$
\lambda^{n-2} \delta \lambda^{2 n}=0
$$

As the proof of [2, Theorem 2], we obtain $\lambda^{n-3} \delta \lambda^{2 n+1}=\lambda^{n-4} \delta \lambda^{2 n+2}=\cdots=\delta \lambda^{3 n-2}=0$. Combining (4) with (5) yields

$$
\lambda^{n-2}[\delta, \lambda] \lambda^{2 n-1}=0 .
$$
Since $\lambda^{n} \delta=0$, we get $\lambda^{n}[\delta, \lambda]=0$. Thus, replacing $\delta$ by $[\delta, \lambda]$ and comparing (4), (5)
and (6), and as the last proof we have

$$
\lambda^{n-3}[[\delta, \lambda], \lambda] \lambda^{2 n-1}=0 .
$$

Continuing in this manner, we finally obtain

$$
\mu \lambda^{2 n-1}=0, \text { where } \mu=\left[\left[\cdots \left[[\delta, \underbrace{\lambda], \lambda], \cdots], \lambda]}_{(n-1)^{\prime} s \lambda} .\right.\right.\right.
$$

Because of $\mu$ is a derivation of $R$, by (8) and Theorem 1 , we get $\mu=0$ or $\lambda^{6 n-4}=$ $\lambda^{3(2 n-1)-1}=0$. If $\lambda^{6 n-4}=0$, then we are done. Assume that $\mu=0$. Thus, as the beginning of the proof, we may suppose that

$$
\nu=\delta \lambda^{n-1}+\lambda \delta \lambda^{n-2}+\cdots+\lambda^{n-1} \delta=0 .
$$


Using (9) repeatedly and $\lambda^{n} \delta=0$, we have $0=\lambda^{n-1} \nu=\lambda^{n-1} \delta \lambda^{n-1}, 0=\lambda^{n-2} \nu \lambda=$ $\lambda^{n-2} \delta \lambda^{n}, \ldots$, and finally we obtain

$$
\delta \lambda^{2 n-1}=0
$$

By Theorem 1 and $\delta \neq 0,(10)$ implies $\lambda^{6 n-4}=\lambda^{3(2 n-1)-1}=0$, as desired.

Chung and Luh [1] showed that in a prime associative ring with characteristic 2 , the nilpotency of nilpotent derivation must be of the form $2^{k}$, where $k \in \mathbb{N}$. Therefore, when $R$ is not 2 -torsion free, the possible values for nilpotency in Theorem 1 and Theorem 2 are further limited. For example, if we assume in Theorem 1 or Theorem 2 that the characteristic of $R$ is $2, \delta \lambda^{21}=0$ and $\delta \neq 0$, or $\lambda^{11} \delta=0$ and $\delta^{2} \neq 0$, then the nilpotency of $\lambda$ must be $1,2,4,8,16$, or 32 .

\section{References}

[1] L. O. Chung and J. Luh, "Nilpotency of derivations II," Proc. Amer. Math. Soc., 91(1984),
357-358. [2] D. W. Jensen, "Nilpotency of derivations in prime rings," Proc. Amer. Math. Soc.,
123(1995), 2633-2636.

[3] E. C. Posner, "Derivations in prime rings," Proc. Amer. Math. Soc., 8(1957), 1093-1100.

[4] C. T. Yen, "Prime ring with a derivation whose some power image is contained in the nucleus," Soochow J. Math., 21(1995), 475-478.

[5] C. T. Yen, Nonassociative rings with skew derivations and rings with associators in the nuclei, Ph. D. thesis, Taiwan University, 1995.

Department of Mathematics, Chung Yuan University, Chung Li, Taiwan, 320, Republic of China. 